\title{
Actions of the Biceps Brachii at the Shoulder: A Review
}

\author{
Dennis Landin ${ }^{\mathrm{a}, \mathrm{b}}$, Melissa Thompson ${ }^{\mathrm{a}}$, Meghan R. Jackson ${ }^{\mathrm{a}}$
}

\begin{abstract}
Bi-articular muscles cross more than one joint and contribute to motion at both joints, and the extremities of the human body contain several such muscles. Actions produced by all muscles are determined, to a large extent, by joint moment arms and muscle length. These are transient factors which change as joint angles are altered. Measuring muscle moments while manipulating both joints will produce a better understanding of the actions of bi-articular muscles. This review summarizes investigations which have explored the actions of the biceps brachii as shoulder and elbow joints are moved into various angle combinations. Clinical implications of the findings are discussed.
\end{abstract}

Keywords: Bi-articular muscles; Joint angles; Moment arms; Scapular plane

\section{Introduction}

The anterior brachial region of the upper extremities contains an important bi-articular muscle, the biceps brachii (BB). Also, lying in this region, but not biarticular are the medially situated coracobrachialis and the anterior, distal brachialis. The brachioradialis arises from the distal brachial region, but its muscle belly lies along the radius, and it is mon-articular.

The BB forms the most prominent contour of the anterior brachial area. It consists of two heads, long and short, that merge into a common belly. The long head arises from the supraglenoid tubercle of the glenoid fossa of the scapula, while the origin of the short head is the coracoid process of the scapula. The tendon of the BB flattens and inserts on the rough posterior aspect of the radial tuberosity. A medially directed expansion of the tendon, the bicipital aponeurosis, fans out across the origin of the forearm flexors on the proximal ulna $[1,2]$.

The actions of the BB at its primary joint have been known for well over 100 years [3]. The BB is a flexor of the elbow,

Manuscript submitted December 26, 2016, accepted January 09, 2017

aSchool of Kinesiology, Louisiana State University, Baton Rouge, LA, USA ${ }^{b}$ Corresponding Author: Dennis Landin, School of Kinesiology, Louisiana State University, 112 Long Fieldhouse, Baton Rouge, LA 70810, USA.

Email: dlandin@1su.edu

doi: https://doi.org/10.14740/jocmr2901w a powerful supinator of the forearm, and has a smaller role in shoulder flexion. This basic description has been relatively unchanged for many years and appears in most anatomy textbooks $[1,4,5]$. However, this description does not consider the influence the second joint may have on the muscle's action at the primary joint, or vice versa [6]. For example, considering the $\mathrm{BB}$ action at the elbow, how does the elbow flexion torque it generates change as the angles of the elbow and shoulder change since muscular insufficiency will ultimately arise? New technology has made it possible to thoroughly explore muscle actions and produce more complete descriptions of muscle actions.

\section{Muscle tissue}

Skeletal muscle tissue comprises about $40 \%$ of the body and consists of parallel bundles of multinucleated cells. This makes the tissue capable of producing considerable power [1, 7]. While this structure is conducive to power, it is hampered by a limited contraction range. But this disadvantage is negated by skeletal lever systems which amplify muscular work [1]. Therefore, when joint angles are optimal, the bi-articular muscles of the extremities can produce considerable torque.

\section{Joint moments}

More precise descriptions of the actions of a bi-articular muscle can be achieved by investigating the joint moments it produces. The moments can be influenced by the muscle's contractile force production and the joint moment arms. Variations in the force production capabilities of muscles influence the potential joint moment production. The force that a muscle can produce is regulated by the level of stimulation it receives, its length when stimulated, and its contraction velocity [8-10]. Joint angles do not alter a muscle's amount of stimulation or its contraction velocity, but do affect a muscle's length and moment arm. The length-tension curve shows that a muscle lengthened or shortened outside the optimal range reduces the power it can generate. A muscle shortened below optimal creates a situation wherein the actin filaments not only fully overlap the myosin filaments, but overlap themselves as well. Lengthening beyond optimal reduces the extent of overlap possible between the actin and myosin filaments $[6,7,11]$.

In regards to moment arms, joint positions will affect the length of moment arms as well as the "moment" itself [12]. A joint moment "is the result of a force acting at a distance from 
the point of motion, or the axis" [6] and the moment arm is the lever that produces the motion around a joint. The greater the moment arm, the greater the joint moment - even if the muscle force remains constant [13].

\section{BB}

There are multiple actions across the elbow and shoulder joints with which the $\mathrm{BB}$ is involved, all in conjunction with other muscles. It is one of three muscles that flex the elbow and it does this work along with the brachialis and brachioradialis $[1,4,5]$. It is also one of three that flex the shoulder (with coracobrachialis and anterior deltoid), and one of two that supinate the forearm (with supinator). Of these motions, the elbow flexion and supination are well established. Its actions at the shoulder have been investigated through a variety of approaches although questions still remain.

Much of the work with the BB has focused on how the long head (BBLH) affects glenohumeral kinematics, stability, etc., and the evidence shows that this activity is significant. Youm et al [14], using cadavers, reported that loading the BBLH led to significant alterations in the muscle's action in the shoulder. Subjecting the BBLH to a force of $22 \mathrm{~N}$, when compared to unloaded specimens, produced significant decreases in glenohumeral range of motion, and the amount of displacement during translations. These findings support those reported years earlier by Pagnani et al [15] and Warner and McMahon [16]. Pagnani et al found that subjecting cadaveric shoulders to a $55 \mathrm{Nm}$ force via the BBLH significantly decreased translation in anterior, inferior, and superior directions. Warner et al had patients with confirmed loss of the proximal attachment of the BBLH tendon move through a wide range of humeral abduction $\left(0,45,90\right.$, and $\left.120^{\circ}\right)$. Relative to the unaffected shoulder they reported a $2-6 \mathrm{~mm}$ increase in superior translation except when the shoulder was in the anatomical position. The authors concluded that the BBLH tendon served as a humeral head stabilizer during abduction in the scapular plane. In contrast, a 2001 report by Levy et al [17] reported a lack of electrical activity in the BBLH during isolated shoulder movements when the elbow and forearm were controlled. Their speculation was that BBLH activity at the proximal attachment was due to a passive role of the tendon or tension brought about through elbow or forearm activity. However, Chalmers et al [18] reported that when the forearm and elbow were immobilized, a load applied to the distal humerus increased BBLH activity as recorded through electromyography (EMG). These authors concluded that the muscle does play a role in glenohumeral motion. In another recent study, Eshuis and De Gast [19] explored how the BBLH affects axial rotation of the humerus. At $0^{\circ}$ of glenohumeral elevation, a load on the biceps tendon caused an increase in internal humeral rotation. This increase ranged from $0^{\circ}$, under a load of $2.25 \mathrm{~N}$, to $23^{\circ}$ at a load of $82.25 \mathrm{~N}$. At or below $45^{\circ}$ of glenohumeral elevation, a load to the biceps tendon can restrict and facilitate internal and external axial humeral rotation, leading to enlarging the rotatory range of motion. Above $45^{\circ}$ of glenohumeral elevation, a load on the biceps tendon restricted internal and external axial hu- meral rotation, actively enhancing joint stability by increasing torsional rigidity.

Myers et al [20], building on the work of Rodosky et al [21], used baseball players with at least 5 years of organized play. EMG data showed that the BB was active at both the elbow and shoulder during the throwing motion. More specifically, the authors found moderate BB activity in the final phases of throwing: late cocking, propulsion, and deceleration. They concluded that the BB may help the shoulder resist torsional forces which reduces the stress placed on the inferior glenohumeral ligament. These findings show that the BB contracts during a wide range of glenohumeral movement, but do not provide evidence that the contribution is significant. In addition, these results do not offer information about how various joint positions may affect the BB moments. Furthermore, much of the research with the BB concerns the effectiveness of various surgical procedures and has not been aimed toward clarifying its actions [22], and consequently is not germane to this review.

A few investigations have explicitly explored how joint angles affect the BB torque production or activation levels. Levy et al [17] measured long head BBLH activity with finewire electrodes while subjects performed a variety of shoulder movements under selected conditions. Trials alternated between weighted and unweighted, at fast and slow speeds, and with the elbow and wrist joints externally stabilized. Specifically, the subjects performed fast and slow flexion, scapular abduction, internal and external rotation all in weighted and unweighted conditions. Their results showed little activity in the BBLH in any of the tested movements. The activity that was noted occurred early in the flexion motion, that is, in the first $30^{\circ}$ of movement, and then when performing a fast flexion movement with 5 pounds of resistance. Furthermore, Levy et al reported that this low level of biceps activity did not change with various elbow and forearm positions, and that it was almost always accompanied by substantial activity in the brachialis. These authors concluded that the BBLH is not active in isolated shoulder movement when the angle of the elbow and forearm are controlled.

In our paper, Landin et al [23], we took a different approach. Rather than recorded the muscle activity during voluntary motions, we externally stimulated the BB and measured the shoulder elevation isometric torque it produced across specific combinations of shoulder and elbow angles. The shoulder angle was controlled by an isokinetic dynamometer (Biodex System 3, Shirley, NY) while the elbow angles were controlled with removable casts. Six shoulder angles $\left(0^{\circ}, 30^{\circ}, 60^{\circ}, 90^{\circ}\right.$, $105^{\circ}$ and $\left.120^{\circ}\right)$ were crossed with four elbow angles $\left(0^{\circ}, 30^{\circ}\right.$, $60^{\circ}$ and $90^{\circ}$ ). At each joint combination, the right $\mathrm{BB}$ of the subjects was externally stimulated following the formula initially devised by Li et al [9]. The electrical stimulations were delivered through surface electrodes placed across the bellies of both heads. Shoulder elevation occurred within the scapular plane. Data were collected on three dependent measures: 1) the passive moment which represented the torque produced in the absence of the external stimulation; 2) maximum moment which was the torque recorded during the stimulation phase; and 3 ) stimulated moment which reflected the torque produced when the contribution of the passive tissue was removed. For 


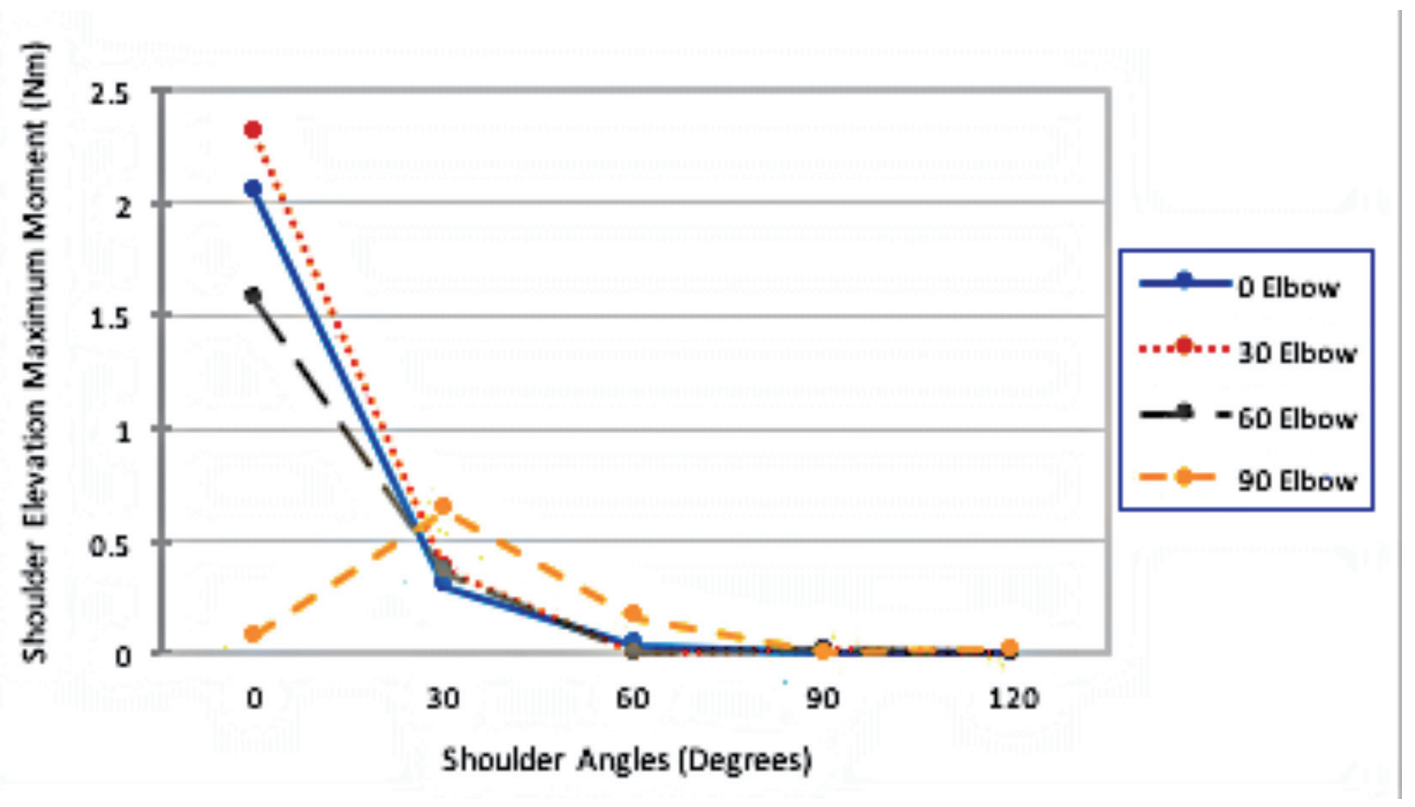

Figure 1. Peak torque at each joint combination.

the purposes of this review, the maximum moment is the most pertinent.

Figure 1 shows the maximum moment recorded at each joint angle combination. Overall, the isometric force was not substantial at any combination indicating that the BBLH does not play a major role in shoulder elevation in the scapular plane. Furthermore, as can be seen, elevating the shoulder to $30^{\circ}$ produced a sharp decline in the isometric force, and at $60^{\circ}$ of shoulder elevation the torque was negligible at all joint combinations. To put this in perspective, in a cadaveric study by Lui et al [24], a shoulder abduction moment of 24.9 $\mathrm{Nm}$ was produced by the supraspinatus, infraspinatus, subscapularis and all three sections of the deltoid. Of this total, the anterior deltoid contribution was reported to be $7.6 \mathrm{Nm}$. Lui et al further reported that the moment arm of the deltoid was quite small at low glenohumeral elevation but increased progressively as elevation increased. Although we used shoulder elevation in the scapular plane, so only partial abduction was involved, our results [23] showed that while the contribution of the BBLH was, in fact, small at low levels of glenohumeral elevation, it becomes nearly non-existent as elevation increases, and as Lui's results suggest, action of the anterior deltoid becomes prominent. Overall, it appears that the BBLH does not contribute much to shoulder elevation, and when it does affect this motion, it is near maximal length [23].

In conclusion, the BBLH is thought to enhance the dynamic stability of the shoulder joint [25-27]. Our 2008 findings [23] support this position but only in the initial $30^{\circ}$ of elevation. Beyond $30^{\circ}$, the BBLH, even though contracting, does not create a noteworthy elevation moment, which suggests that it cannot serve as a dynamic shoulder stabilizer in higher ranges of elevation. However, the BBLH can be injured in throwing motions and as we argued in 2008, this is most likely in the deceleration phase, which would go through the $30^{\circ}$ position. Based on the current evidence, exercises planned to rehabilitate the shoulder should involve low level elevation, which can increase the BBLH dynamic role in stabilizing the joint when elevated to $30^{\circ}$ or less. Furthermore, incorporating eccentric contractions through the lower range of elevation would be appropriate since this could mimic the deceleration phase of the overhead throwing motion.

\section{References}

1. Salmons S. Muscle. In: Williams PL, Bannister LH, Berry MM, Collins P, Dyson M, Dussek JE, Ferguson MWJ, editors. Gray's anatomy. New York: Churchill Livingstone; 1995. p. 737-900.

2. Moore KL, Dalley AF. Clinically Oriented Anatomy. Philadelphia: Lippincott, Williams \& Wilkins: 1996; 615616.

3. Pickering T, Howden R. Muscle. Grays' anatomy, 15th edition. Facsimile Reproduced by Barnes \& Nobles, 1995.

4. Tortora GJ, Nielsen MT. Principles of human anatomy. Hoboken, NJ: Wiley \& Sons; 2017.

5. Van De Graaff KM. Human Anatomy. Dubuque, IA: McGraw-Hill; 2002.

6. Houglum PA, Bertoti DB. Brunnstrom's Clinical Anatomy. Philadelphia, PA: FA Davis; 2012.

7. Guyton AC, Hall JE. Textbook of Medical Physiology, 9th ed. WB Saunders; Philadelphia, 1996.

8. Buford WL, Jr., Ivey FM, Jr., Malone JD, Patterson RM, Peare GL, Nguyen DK, Stewart AA. Muscle balance at the knee - moment arms for the normal knee and the ACLminus knee. IEEE Trans Rehabil Eng. 1997;5(4):367379.

9. Li L, Landin D, Grodesky J, Myers J. The function of gastrocnemius as a knee flexor at selected knee and ankle 
angles. J Electromyogr Kines. 2002;12:385-390.

10. Rassier DE, MacIntosh BR, Herzog W. Length dependence of active force production in skeletal muscle. J Appl Physiol (1985). 1999;86(5):1445-1457.

11. Gordon AM, Huxley AF, Julian FJ. The variation in isometric tension with sarcomere length in vertebrate muscle fibres. J Physiol. 1966;184(1):170-192.

12. Winters JM, Kleweno DG. Effect of initial upper-limb alignment on muscle contributions to isometric strength curves. J Biomech. 1993;26(2):143-153.

13. Bahler AS. Series elastic component of mammalian skeletal muscle. Am J Physiol. 1967;213(6):1560-1564.

14. Youm T, ElAttrache NS, Tibone JE, McGarry MH, Lee TQ. The effect of the long head of the biceps on glenohumeral kinematics. J Shoulder Elbow Surg. 2009;18(1):122-129.

15. Pagnani MJ, Deng XH, Warren RF, Torzilli PA, O'Brien SJ. Role of the long head of the biceps brachii in glenohumeral stability: a biomechanical study in cadavera. J Shoulder Elbow Surg. 1996;5(4):255-262.

16. Warner JJ, McMahon PJ. The role of the long head of the biceps brachii in superior stability of the glenohumeral joint. J Bone Joint Surg Am. 1995;77(3):366-372.

17. Levy AS, Kelly BT, Lintner SA, Osbahr DC, Speer KP. Function of the long head of the biceps at the shoulder: electromyographic analysis. J Shoulder Elbow Surg. 2001;10(3):250-255.

18. Chalmers PN, Cip J, Trombley R, Cole BJ, Wimmer MA, Romeo AA, Verma NN. Glenohumeral Function of the Long Head of the Biceps Muscle: An Electromyographic Analysis. Orthop J Sports Med. 2014;2(2):2325967114523902.

19. Eshuis R, De Gast A. Role of the long head of the biceps brachii muscle in axial humeral rotation control. Clin Anat. 2012;25(6):737-745.

20. Myers JM, Lephart SM, Rodosky MW, Ju YY, Fu F. Role of The Biceps Brachii During Throwing: An EMG Study. Paper presented at the 49th Annual Meeting of the Orthopaedic Research Society. Feb. 2003, New Orleans, LA.

21. Rodosky MW, Harner CD, Fu FH. The role of the long head of the biceps muscle and superior glenoid labrum in anterior stability of the shoulder. Am J Sports Med. 1994;22(1):121-130.

22. Berlemann U, Bayley I. Tenodesis of the long head of biceps brachii in the painful shoulder: improving results in the long term. J Shoulder Elbow Surg. 1995;4(6):429435.

23. Landin D, Myers J, Thompson M, Castle R, Porter J. The role of the biceps brachii in shoulder elevation. J Electromyogr Kinesiol. 2008;18(2):270-275.

24. Liu J, Hughes RE, Smutz WP, Niebur G, Nan-An K. Roles of deltoid and rotator cuff muscles in shoulder elevation. Clin Biomech (Bristol, Avon). 1997;12(1):32-38.

25. Kido T, Itoi E, Konno N, Sano A, Urayama M, Sato K. Electromyo- graphic activities of the biceps brachii during arm elevation in shoulders with rotator cuff tears. Acta Ortho Scand. 1998;69(6):575-579.

26. Kido T, Itoi E, Konno N, Sano A, Urayama M, Sato K. The depressor function of biceps brachii on the head of the humerus in shoulders with tears of the rotator cuff. J Bone Joint Surg (Br). 2000;82(3):416-419.

27. Paxinos A, Walton J, Tzannes A, Callanan M, Hayes K, Murrell GA. Advances in the management of traumatic anterior and atraumatic multidirectional shoulder instability. Sports Med. 2001;31(11):819-828. 